\title{
Physics prospects with the ALFA and AFP detectors
}

\author{
Petr Hamal ${ }^{* \dagger}$ \\ Palacky University, Faculty of Science, RCPTM, Joint Laboratory of Optics of Palacky \\ University and Institute of Physics of the Academy of Sciences of the Czech Republic, \\ 17. listopadu 12, Olomouc, 771 46, Czech Republic \\ E-mail: petr.hamaldcern.ch
}

\begin{abstract}
We will describe the experimental challenge presented by the measurement of the forward protons at the LHC. The ATLAS ALFA and AFP projects involve the deployment of detectors capable of detecting protons that emerge intact from the interaction with very small scattering angles. In the case of the ALFA detector, located at $\pm 240 \mathrm{~m}$ from the ATLAS interaction point, this measurement has to be performed using special high $\beta^{*}$ beam optics. The detector chosen for the task is a nearly edgeless scintillating fibre tracker capable of making measurements at very low $t$ - below $10^{-3} \mathrm{GeV}^{2}$ - in the nuclear Coulomb interference region. In order to reach these very small $t$ values we need to deploy the detector very near to the beam line utilizing Roman Pots. The main purpose of the ALFA detector is to measure the total p-p cross-section, a fundamental quantity in hadronic physics of importance to particle and to cosmic ray physicists and to provide absolute measurement of luminosity which includes eventual measurements of dependency of nuclear slope $b$ and $\rho$ parameters on $t$. Another capability of the ALFA detector is measurement of soft diffraction and some exclusive processes. The main purpose of the AFP detector is to measure exclusive physics processes and anomalous couplings. The AFP detectors are placed at \pm 204 and \pm 212 meters on both sides of the ATLAS experiment, giving ATLAS the remarkable capability to tag and measure, with 3-D silicon detectors, both protons in exclusive central diffractive processes. The use of precision timing detectors allows this type of physics to be pursued to high luminosity for the first time. The AFP project opens up an important new window on LHC physics not available with the standard ATLAS detector. In this talk the experimental challenges in constructing this detector as well as the status of the project will be discussed.
\end{abstract}

International Conference on the Structure and the Interactions of the Photon including the 20th International Workshop on Photon-Photon Collisions and the International Workshop on High Energy Photon Linear Colliders

20 - 24 May 2013

Paris, France

\footnotetext{
* Speaker.

${ }^{\dagger}$ on behalf of Forward Detector Group of ATLAS collaboration.
} 


\section{INTRODUCTION}

We will describe the experimental challenge presented by the measurement of the forward protons at the LHC. The ATLAS ALFA (running) and AFP (under approval) projects involve the deployment of detectors capable of detecting protons that emerge intact from the interaction with very small scattering angles. Both detectors belong to the ATLAS Forward Detectors group. The ALFA detectors are located at $\pm 237 \mathrm{~m}$ and $\pm 241 \mathrm{~m}$ from the ATLAS interaction point (IP). Since 2011 all four ALFA stations measure and take data mostly during special LHC high $\beta^{*}$ runs but not only (the ATLAS heavy ion runs 2013). The actual plan is to build AFP detectors at $\pm 204 \mathrm{~m}$ and $\pm 212 \mathrm{~m}$ from the ATLAS interaction point. If the AFP project is approved the installation should start in the LHC winter shutdown 2015. Activities of these two detectors are described in the next sections.

The rest of the ATLAS Forward Detectors group consists of the Cherenkov light detectors (LUCID) at $\pm 17 \mathrm{~m}$ from the IP and the Zero-Degree Calorimeters (ZDC) detectors at $\pm 140 \mathrm{~m}$ from the IP. LUCID is primarily dedicated to online luminosity monitoring. It is a relative luminosity detector covering $5.61<|\eta|<5.93$. Its main purpose is to detect inelastic proton-proton scattering in the forward direction, in order to both measure the integrated luminosity and to provide online monitoring of the instantaneous luminosity and beam conditions [W]. The primary purpose of the ZDC is to detect forward neutrons and photons with $|\eta|>8.3$ in both proton-proton and heavy-ion collisions. For heavy ion collisions the ZDC's play a key role in determining the centrality of such collisions, which is strongly correlated to the number of very forward (spectator) neutrons [D].

\section{ALFA}

The measurement of intact forward protons is performed using special high $\beta^{*}$ beam optics and low luminosity runs [ [ 4 ]. This is due to a low ALFA radiation hardness and due to an elimination of vertex smearing.

The detector chosen for the task is a nearly edgeless scintillating fibre tracker capable of making measurements at very low $t$ - below $10^{-3} \mathrm{GeV}^{2}$ - in the nuclear Coulomb interference region. In order to reach these very small $t$ values we need to deploy the detector very near to the beam line utilizing Roman Pots. The main purpose of the ALFA detector is to measure the total protonproton cross-section, a fundamental quantity in hadronic physics of importance to particle and to cosmic ray physicists and to provide absolute measurement of luminosity which includes eventual measurements of dependency of nuclear slope $b$ and $\rho$ parameters on $t$. Another capability of the ALFA detector is measurement of soft diffraction and some exclusive processes.

\subsection{Detector description}

The ALFA is a tracking detector. It is designed to measure the position of the elastically scattered protons coming from the ATLAS interaction point under a very small angle. For this measurement the ALFA uses Roman Pot technology with vertical movement. This technology allow us to come very close to the beam (up to $1 \mathrm{~mm}$ from the centre of ATLAS beam pipe).

The whole detector consists of two parts. The first part, so-called a main part, is the Main Detector (MD). The MD consists of 10 titanium plates with two layers of fibres glued under $+45^{\circ}$ or 
$-45^{\circ}$. Each layer contains 64 scintillating squared fibres $\left(0.5 \times 0.5 \mathrm{~mm}^{2}\right)$. To increase a total spatial resolution all 10 plates are staggered along a horizontal axis. A spatial resolution of this system (in $x$ and $y$ axis) is about $30 \mu \mathrm{m}$. The MD is radiation hard up to $300 \mathrm{kRad}(1 \mathrm{rad}=0.01 \mathrm{~Gy}$ ) which is enough for low- $\mu$ runs data taking. The $\langle\mu\rangle$ is a mean value of number of interactions during a collision. For the low- $\mu$ runs the $\langle\mu\rangle$ is below 1 , for the standard LHC runs the $\langle\mu\rangle$ is usually in the range $\langle\mu\rangle \in(20,40)$.

The second part of the ALFA are so-called the Overlap Detectors (OD). These detectors are situated on both sides of the ALFA. They are made by 3 titanium plates with 30 vertically placed scintillating fibres. They serve to relatively align the upper and lower ALFA detectors. The obtained precision of this alignment is $\sim 10 \mu \mathrm{m}$ in horizontal and $\sim 80 \mu \mathrm{m}$ in vertical plane.

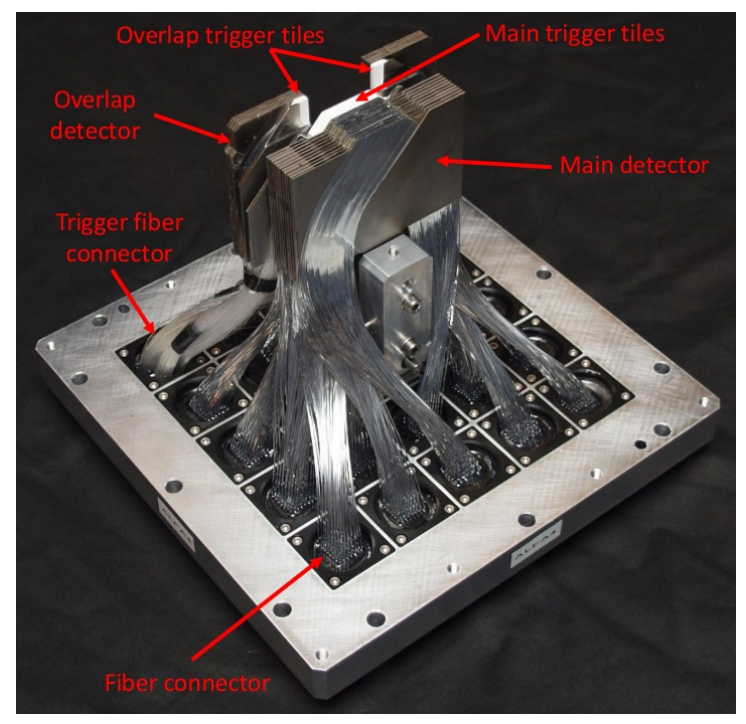

Figure 1: ALFA detector - the support with the main detector, overlap detectors, trigger tiles and fibres connected to readout electronics [B]].

\subsection{Physics}

The number of elastically scattered protons is related to the total interaction rate through the optical theorem [四]

$$
\sigma_{\mathrm{tot}}=4 \pi \cdot \mathfrak{I m}\left[f_{\mathrm{el}}(t=0)\right],
$$

where $\sigma_{\mathrm{tot}}$ is the total cross-section, $f_{\mathrm{el}}$ is the forward elastic scattering amplitude, and $t$ is the standard Mandelstam variable. This theorem states that the total cross section is directly proportional to the imaginary part of the forward scattered elastic amplitude extrapolated to zero momentum transfer. If $p$ is the 3 -momentum of the scattered proton, then for small values of scattering angle $\theta$ one can write

$$
-t=(p \theta)^{2} \text {. }
$$

If we consider a relatively small $t$ value the rate of elastic scattering events can be written as

$$
\frac{\mathrm{d} N}{\mathrm{~d} t}=L \pi\left|f_{\mathrm{C}}+f_{\mathrm{N}}\right|^{2} \approx L \pi\left|-\frac{2 \alpha_{\mathrm{EM}}}{|t|}+\frac{\sigma_{\mathrm{tot}}}{4 \pi}(\imath+\rho) \exp \left(\frac{-B|t|}{2}\right)\right|^{2},
$$


where $f_{\mathrm{C}}$ corresponds to the Coulomb, $f_{\mathrm{N}}$ to the nuclear interaction amplitude and

$$
\rho=\left.\frac{\mathfrak{R e} f_{\mathrm{el}}}{\mathfrak{I m} f_{\mathrm{el}}}\right|_{t \rightarrow 0} .
$$

For simplicity, we have excluded the proton form factor, and there are also other corrections that should be included in the final analysis.

The most suitable method to perform the ALFA measurements is to use so-called "parallel-topoint" optics. In this type of optics the betatron oscillation between the IP of the elastic collision and the detector position has a $90^{\circ}$ phase difference (only in the vertical plane). In this case all particles scattered at the same angle are, ideally, focused on the same point at the detector, independently of the the interaction vertex position. Thus, a transverse position measurement at the detector gives directly an angular measurement at the interaction point.

The high $\beta^{*}$ optics allows us to access low $t$ values for elastically scattered protons (see Fig. (2)). The $\beta^{*}=90 \mathrm{~m}$ allows mainly total cross section measurements, $\beta^{*}=1000 \mathrm{~m}$ will probably allow to investigate the nuclear Coulomb interference region. In a future, when the ALFA will measure with the highest accessible $\beta^{*}$ in the range $\sim 2 \mathrm{~km}$, it could allow the precise determination of the absolute luminosity, as the region where the scattering amplitude is dominated by the QED will be reached.

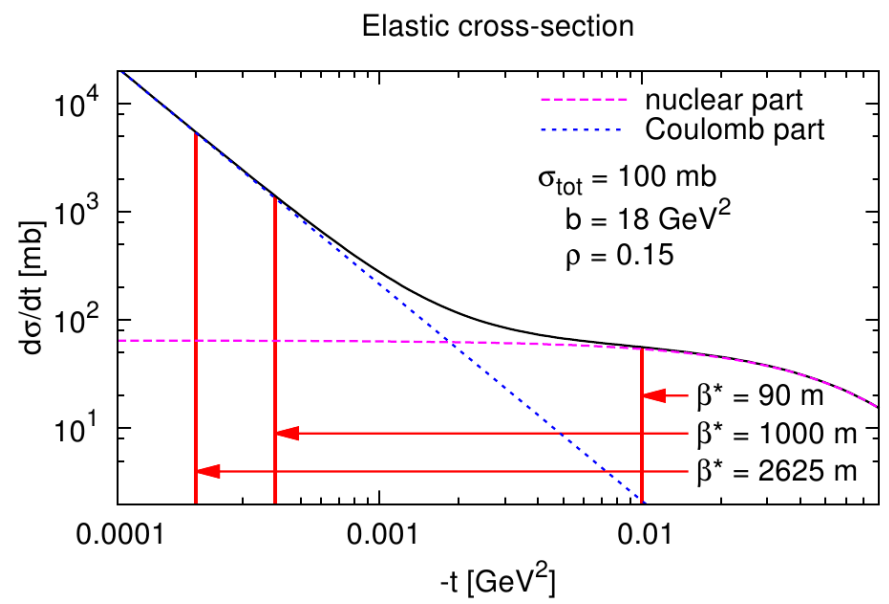

Figure 2: The elastic cross section as a function of $t$ for a possible set of parameters at LHC energies. An estimate of the expected $t$ value reachable at a given $\beta^{*}$ is plotted [5].

Except the absolute luminosity and the total cross section measurement with the elastically scattered protons ALFA detectors can detect diffractive protons. This potentially allow us to study exclusive productions in ATLAS, like $p p \rightarrow p p \pi^{+} \pi^{-}$[四] (the only measurements of exclusive pion production were performed at $62 \& 63 \mathrm{GeV}$ by ABCDHW Collaboration, ISR [ [ 8 , 团]).

\subsection{Summary}

During the LHC operation there were several proton-proton runs dedicated to high- $\beta$ runs and a few proton-Pb runs, where the ALFA detectors were inserted into the LHC beampipe. Except for a few test runs there were three main high- $\beta$ runs and one proton- $\mathrm{Pb}$ run when the ALFA detectors collected data: 
- $\beta=90 \mathrm{~m}$, with the centre of mass energy $\sqrt{ } s=7 \mathrm{TeV}$ (October 2011),

- $\beta=90 \mathrm{~m}$, with the centre of mass energy $\sqrt{ } s=8 \mathrm{TeV}$ (July 2012),

- $\beta=1000 \mathrm{~m}$, with the centre of mass energy $\sqrt{ } s=8 \mathrm{TeV}$ (October 2012).

- $\mathrm{p}-\mathrm{Pb}$ run with the centre of mass energy $\sqrt{ } s=2.75 \mathrm{TeV}$ (January/February 2013).

One example of the physics analysis for $\beta=90 \mathrm{~m}$ and $\sqrt{ } s=7 \mathrm{TeV}$ is shown on Fig. [1]. The plots show reconstructed scattering angle correlation between left and right side of ATLAS IP for elastic candidates after background rejection cuts. The scattering angle is the main variable which is measured with ALFA detectors. The data used in this analysis were taken in the October 2011. After background rejection we obtained about 800k clean elastic events.
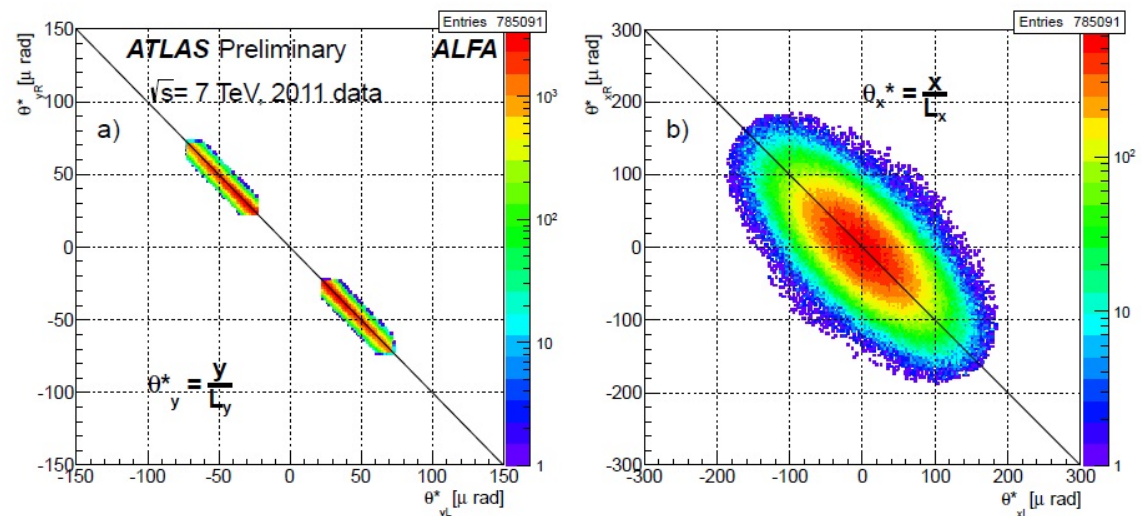

Figure 3: Reconstructed scattering angle correlation between $\mathrm{A}\left(\theta_{\mathrm{xL}}^{*}, \theta_{\mathrm{yL}}^{*}\right)$ and $\mathrm{C}\left(\theta_{\mathrm{xR}}^{*}, \theta_{\mathrm{yR}}^{*}\right)$ side for elastic candidates after background rejection cuts a) in the vertical and $b$ ) in the horizontal plane [ 6 ].

\section{AFP}

The ATLAS Forward Proton (AFP) project is a planned (but still not approved) successor of the ALFA and foreseen for forward diffractive physics at highest LHC intensities. The main goal of the AFP is to measure exclusive physics processes and anomalous coupling. The AFP detectors should be placed at \pm 204 and \pm 212 meters $^{1}$ on both sides of the ATLAS experiment, giving ATLAS the remarkable capability to tag and measure, with 3-D silicon detectors, both protons in exclusive central diffractive processes [प]]. The use of precision timing detectors allows this type of physics to be pursued to high luminosity for the first time. The AFP is designed to run with high pile up. In contrast to the ALFA, the AFP can run during a standard high luminosity run. The AFP project opens up an important new window on LHC physics not available with the standard ATLAS detector.

\footnotetext{
${ }^{1}$ AFP were originally designed to place them at $\pm 206 \mathrm{~m}$ and $\pm 214 \mathrm{~m}$ from the ATLAS IP but it was decided to move them $2 \mathrm{~m}$ closer to the ATLAS IP.
} 


\subsection{Detector description}

The AFP should contains two kinds of detectors: a tracker detector and a time-of-flight (TOF) detector. The main purpose of the 3-D silicon pixel tracker is a measurement of the trajectory of beam collision deflected protons coming from the ATLAS interaction point. It should consist of 5 or 6 silicon pixel layers and should use the FE-I4 readout chip. A distance of the edge of the detector from a centre of the beam should be up to $1.5 \mathrm{~mm}$. The spatial resolution should be around $10 \mu \mathrm{m}$ in the horizontal plane and about $30 \mu \mathrm{m}$ in the vertical plane.

The second type of AFP detector is a timing detector. The aim of the time-of-flight detector is to measure with a precision of $\sim 10$ ps or better to reject pile-up background. Other requirements on the timing detector, includes the high efficiency and the full acceptance, the high rate capability, the segmentation from the multi-proton timing, the L1 trigger capability, the robust operation in the high radiation environment. To achieve the required time resolution the detector consists of $4 \times 8$ $\left(5 \times 5 \mathrm{~mm}^{2}\right)$ quartz bars, $8-12 \mathrm{~cm}$ long ( 8 measurements per detector with $30-40$ ps resolution each). The proton is deflected into one of the rows and measured by eight different bars/detector with a micro-channel plate photomultiplier.

\subsection{Physics}

The purpose of the new forward detector is a possibility to identify and record events with leading intact protons emerging from collisions occurring in ATLAS. The main physics output comes from the remarkable capability of AFP to tag and measure both protons in the final state. The AFP physics program [12] focuses on

- exclusive physics processes - (eg. jets, $\gamma \gamma, \mathrm{W} / \mathrm{Z}$ ),

- anomalous couplings studies $(\gamma \gamma \mathrm{WW}, \gamma \gamma \mathrm{ZZ}, \gamma \gamma \gamma \gamma)$,

- SUSY,

- magnetic monopoles,

- any production of new objects (with mass up to $2 \mathrm{TeV}$ ) via photon or gluon exchanges: Kaluza-Klein resonances, black holes, etc.

With the AFP detectors it will be possible to measure the mass of a produced object inde-

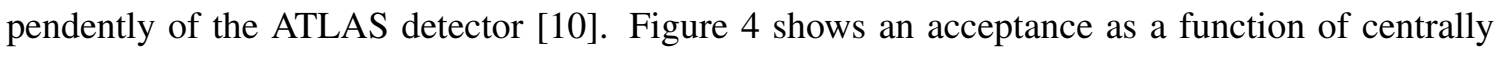
produced mass for $210+210 \mathrm{~m}$ proton tags at different positions of the tracking detector relative to the beam.

\section{SUMMARY}

The ALFA detectors have passed successfully measurements with $\beta^{*}=90 \mathrm{~m}$ at $\sqrt{ } s=7 \mathrm{TeV}$ and $\beta^{*}=1000 \mathrm{~m}$ at $\sqrt{ } s=8 \mathrm{TeV}$. For the future there is a clear program to achieve the highest accessible $\beta^{*}$ (in the range $\sim 2 \mathrm{~km}$ ). The physics analysis of data for $\beta^{*}=90 \mathrm{~m}$ optics is finishing, the analysis of data for $\beta^{*}=1000 \mathrm{~m}$ optics is in progress. In parallel with data analysis there are also programs for a better understanding of LHC optics for special high $\beta^{*}$ runs, for a cooling 


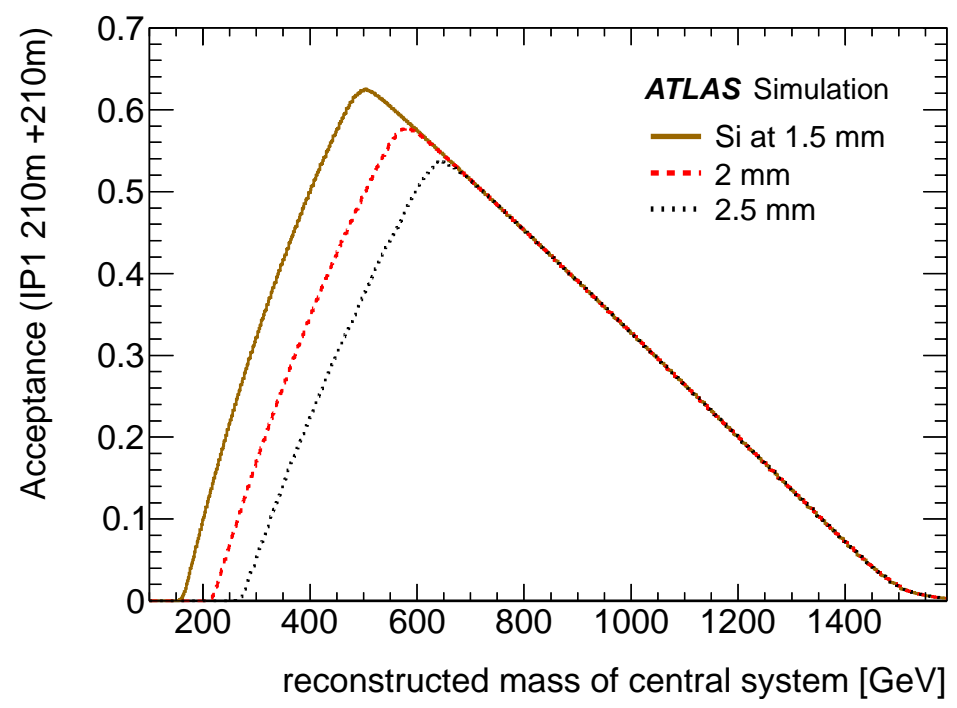

Figure 4: Acceptance as a function of centrally produced mass for $210+210 \mathrm{~m}$ proton tags for the edge of the silicon detector active region located at different distances from the beam [U]].

upgrade of the ALFA detectors or the program of moving neighbouring stations closer for larger $\theta$ angle.

The AFP project is still before ATLAS approval. It needs to be supported by the physics program demonstrated by a set of simulations. There is a very good progress of the AFP model integration in ATLAS full simulation studies. The timing detectors are still under development (several variants are possible). A prototype of the fast timing system reached $\sim 24$ ps resolution [3]. This prototype was validated with beam and laser tests. The next step is to conclude which movable system will be used for AFP detectors.

\section{ACKNOWLEDGMENTS}

The author gratefully acknowledges the support of the Ministry of Education of the Czech Republic Project No. LG 13009, International Experiment ATLAS-CERN.

This work was supported by the student project PrF_2013_006 of the Palacky University.

Copyright CERN for the benefit of the ATLAS collaboration.

\section{References}

[1] ATLAS Collaboration, Lucid Detector Description at https://twiki.cern.ch/twiki/bin/viewauth/Atlas/LucidDescriptio[cited July, $1^{\text {st }} 2013$ ]

[2] ATLAS Collaboration, Zero Degree Calorimeters for ATLAS, CERN-LHCC-2007-001

[3] S. Jakobsen, Technical and operational overview of ATLAS-ALFA, ATL-FWD-INT-2013-002

[4] ATLAS Luminosity and Forward Physics Community, ATLAS TDR 018, CERN/LHCC/2008-004 
[5] M. Trzebinski, on behalf of the ATLAS Collaboration, Towards a Total Cross Section Measurement with the ALFA Detector at ATLAS

[6] ATLAS Collaboration, Public Forward Detector Plots for Collision Data at https: //twiki.cern.ch/twiki/bin/view/AtlasPublic/ForwardDetPublicResults [cited July, $1^{\text {st }}$ 2013]

[7] P. Lebiedowicz, A. Szczurek, Exclusive $p p \rightarrow p p \pi^{+} \pi^{-}$reaction: from the threshold to LHC, Phys. Rev. D81 (2010) 0360

[8] A. Breakstone et al. (ABCDHW Collaboration), Inclusive pomeron-pomeron interactions at the CERN ISR, Z. Phys. C42 (1989) 387, Erratum-ibid. C43 (1989) 522 IS-J-3226

[9] A. Breakstone et al. (ABCDHW Collaboration), The Reaction pomeron-pomeron $->\pi^{+} \pi^{-}$and an unusual production mechanism for the $f_{2}(1270)$, Z. Phys. C48 (1990) 569-576 IS-J-4087

[10] ATLAS Collaboration, ATLAS Letter of Intent Phase-I Upgrade, CERN LHCC-2011-012, LHCC-I-020

[11] O. Kepka, C. Royon, L. Schoeffel, R. Staszewski, M. Trzebinski, R. Zlebcik, Physics Cases within the AFP project, ATL-COM-PHYS-2012-775

[12] O. Kepka, C. Royon, AFP physics topics, ATL-COM-PHYS-2013-390

[13] I. Howley, A. Brandt for AFP Timing Group, T-958' Fast Timing $R \& D$ at http://www.fnal.gov/directorate/program_planning/all_experimenters_ meetings/special_reports/Howley_T958_01_23_12.pdf [cited July, ${ }^{\text {st }}$ 2013] 